\title{
Actualización de modelos de demanda con datos reducidos. El caso de Riad
}

\author{
Enrique Lillo López \\ Diadro Consulting SL
}

\begin{abstract}
RESUMEN
Las encuestas domiciliarias de movilidad (EDM) son un instrumento valioso para la planificación de transportes, y la principal fuente de datos para el ajuste de modelos de demanda en las grandes ciudades. Por los recursos necesarios (económicos y organizativos) y el plazo para su realización y explotación, la actualización de los modelos con nuevas EDM no es siempre posible, por más que cambien las condiciones de la ciudad (nueva población, nuevos desarrollos, etc.) o los patrones de movilidad.
\end{abstract}

Sin embargo, partiendo de la información de una EDM previa, es posible actualizar y mejorar los modelos de demanda con un coste en recursos y tiempo razonable; realizando encuestas domiciliarias con una muestra más reducida y seleccionada para cumplir con los objetivos previamente marcados para la actualización de los modelos de demanda.

En esta ponencia se presenta una experiencia de este tipo realizada para actualizar los modelos de demanda de Riad (Arabia Saudí) por encargo de Arriyadh Development Authority (ADA). El proceso se realizó entre los años 2014-2015.

\section{INFORMACIÓN GENERAL}

\subsection{La ciudad}

Riad es la capital el reino de Arabia Saudí, y está situada en el centro de la península Arábiga. Es una gran urbe de más de 7.3 millones de habitantes, y ha experimentado un gran crecimiento en los últimos años; y que es previsible que se mantenga por las actuaciones urbanísticas previstas, como el nuevo Centro Financiero, que aspira a ser el más importante de Oriente Medio.

A pesar de su tamaño y población, el transporte está prácticamente monopolizado por el automóvil. Existen pocas líneas oficiales de transporte público y tanto la frecuencia de paso como el estado del material rodante hacen poco atractivo su uso. Alternativamente y para servir a parte de la población sin acceso al vehículo privado, muchas empresas tienen flotas de autobuses para transportar a sus trabajadores; y también existe un transporte más o menos informal con minibuses con itinerarios desde los barrios populares hasta los destinos de trabajo más comunes. Además, hay una gran flota de taxis.

Como consecuencia de lo anterior, el porcentaje de viajes en vehículo privado supone el $84 \%$ del total (incluidos los viajes a pie), y la congestión es el estado habitual de gran parte de las vías de Riad durante gran parte del día. Este uso intensivo del automóvil está 
favorecido por el precio del combustible para los usuarios, que es uno de los más bajos del mundo (0.2 €/litro en 2016).

\subsection{Nuevo plan de transporte para Riad}

La Administración, a través de ADA desarrolló un ambicioso plan de transporte púbico para Riad, que comprendía la construcción de 6 líneas de metro con $175 \mathrm{~km}$, y una extensa red de autobuses convencionales junto con varios corredores de BRT que totalizan $85 \mathrm{~km}$. Las obras del metro iniciaron en 2014 y está prevista su apertura el año 2018.

El diseño y el dimensionamiento inicial de las líneas de metro y autobús se realizaron con el modelo de demanda existente anteriormente en Riad, del año 2004. Sin embargo, la Administración entendió que el modelo previo no reflejaba correctamente el estado de la movilidad actual en Riad, y además, presentaba algunos problemas que exigían su actualización para analizar con más detalle el impacto de la nueva red de transporte público y otras actuaciones en la red viaria previstas para entrar en operación a corto plazo (20162018).

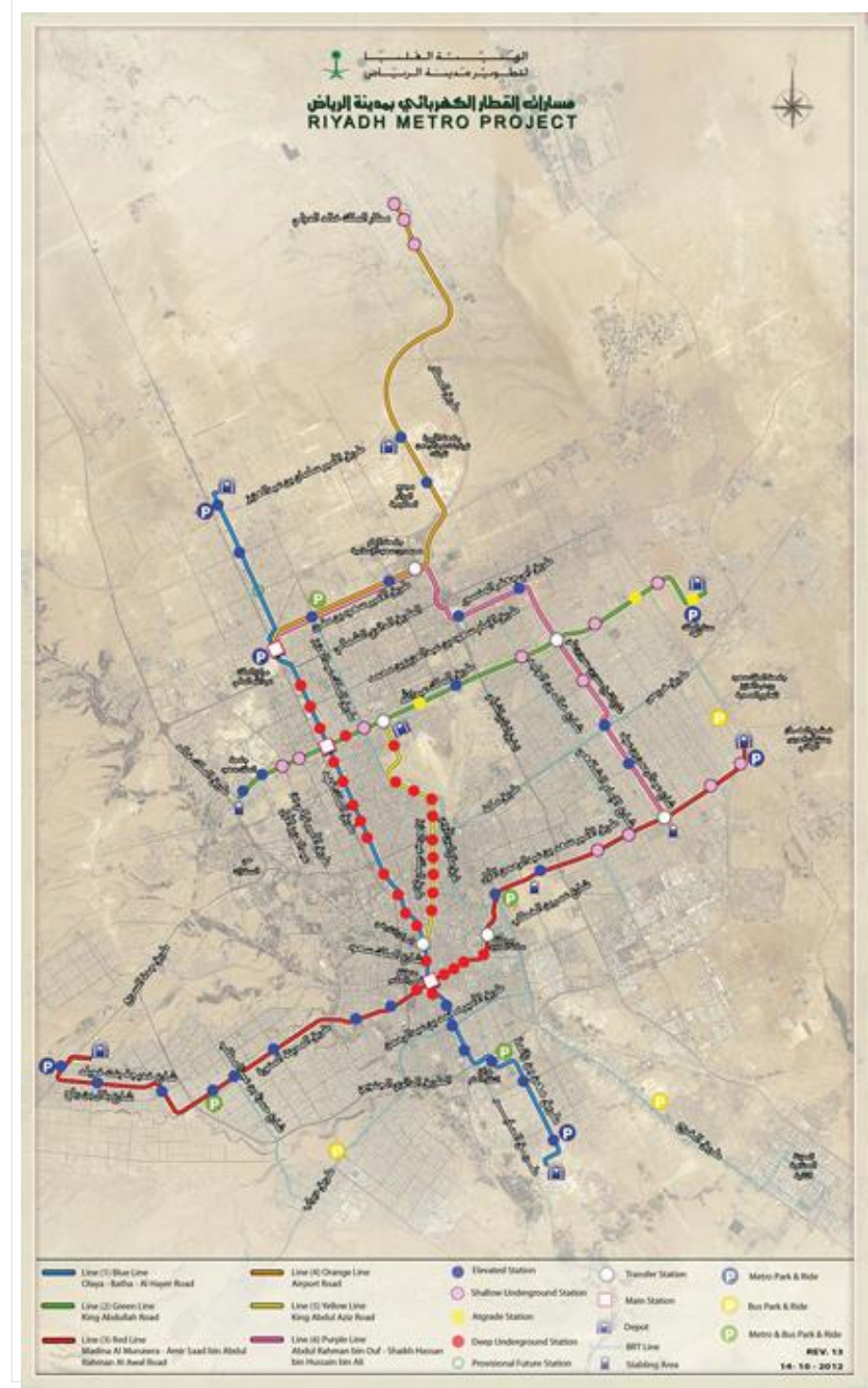




\section{Figura 1 - Red de metro en construcción en Riad}

Paradójicamente, a pesar del volumen de inversión previsto para la construcción de los nuevos metros y líneas de autobús, ADA estaba limitada tanto financieramente como por el plazo de realización para actualizar los datos y los modelos con una nueva EDM; por lo que decidió plantear una actualización de los modelos con el menor uso de encuestas posible; pero que al mismo tiempo pudiera dar respuesta a las principales inquietudes acerca del nuevo plan:

- Número de usuarios de las nuevas líneas de metro y autobús

- Dimensionamiento de los servicios

- Ingresos del sistema

- Impacto en la red de vehículo privado

- Efecto de las medidas disuasorias de utilización del vehículo privado

\subsection{Modelo de demanda 2004}

El modelo del año 2004 era un modelo de cuatro etapas, que estaba basado, entre otras fuentes, en una EDM, y estaba implementado en la plataforma EMME. El modelo estimaba en primer lugar las matrices diarias, y las matrices para la hora punta y los períodos valle se derivaban de las matrices diarias.

Una de las fuentes de incertidumbre acerca de los resultados era el número de factores de ajuste que se utilizaban para calcular las matrices horarias finales. En muchos casos, estos factores eran mayores que 2; lo que indicaba que los modelos explicaban menos de la mitad de los viajes; y el resto se estimaban por diferencia con los aforos en la red viaria.

Un primer análisis de los datos mostró sesgos importantes en la EDM 2004, que podían explicar la necesidad de factores de ajuste tan altos. En efecto, observamos que el número de viajes por persona decrecía significativamente a medida que el tamaño familiar crecía, pasando de 2.68 viajes/persona/día para los hogares unipersonales a 0.47 viajes/persona/día para los hogares de más de 15 personas, como se indica en la figura siguiente. 


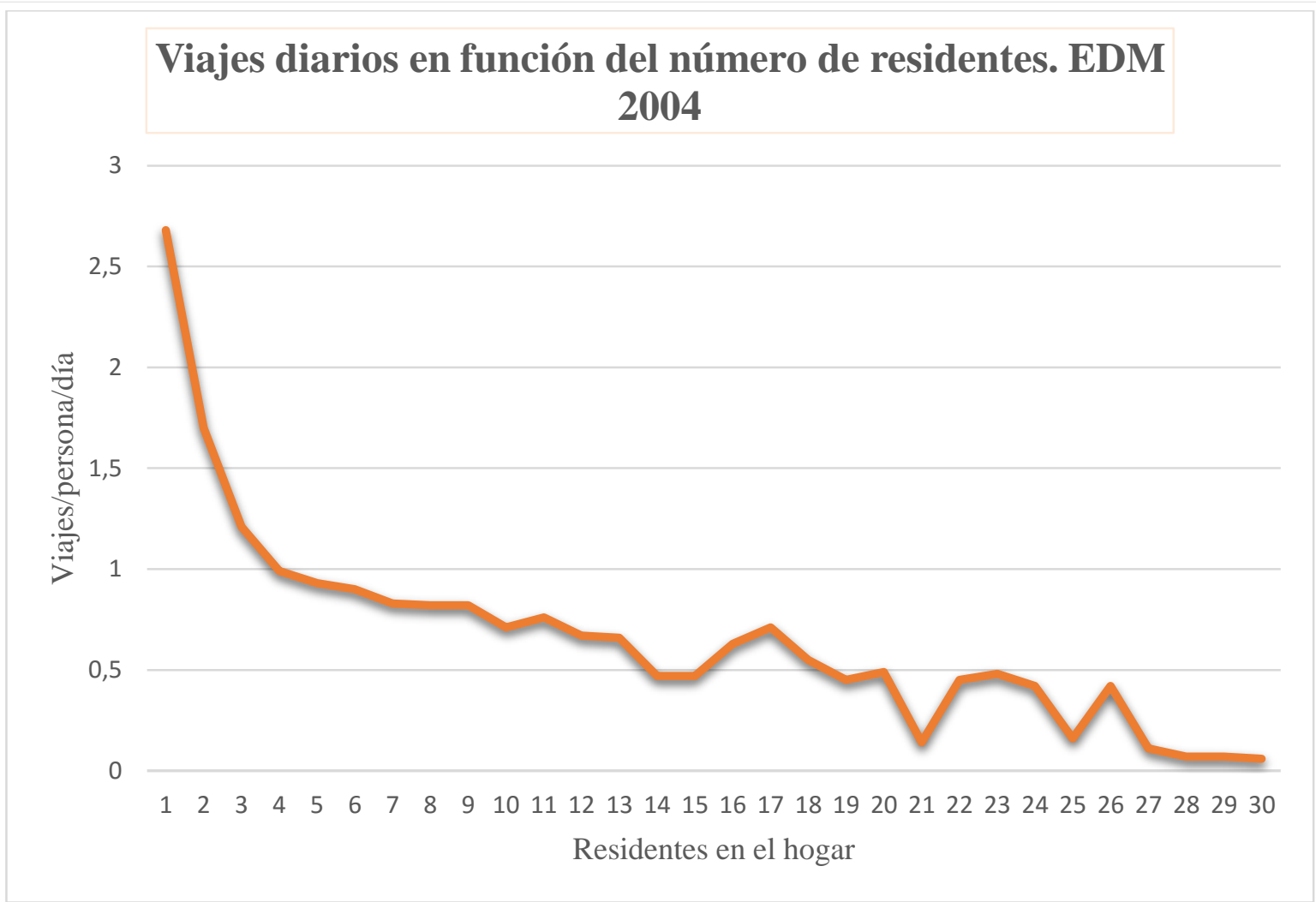

Figura 2 - Viajes por persona EDM 2004

Este sesgo refleja evidentes problemas en la realización de la encuesta anterior, sesgos que pueden tener una explicación por las particularidades de la sociedad saudí. En efecto, para un encuestador no es posible acceder a todos los miembros de la familia, y normalmente sólo se puede contactar con el cabeza de familia. Finalmente, el cabeza de familia rellena los datos de los viajes de todos los miembros de la familia, y sólo registra algunos; de manera que los únicos fiables son los de los hogares unipersonales.

Además, los modelos presentaban otros serios problemas que hacían conveniente su revisión y eventualmente, su sustitución:

Falta de representación en la muestra de importantes segmentos de la población, como los residentes no saudíes

- Muestras muy pequeñas en algunas zonas de transporte, especialmente en aquellas de población con menos ingresos

- El modelo de producción de viajes era un modelo de categoría de hogares, lo que hace que su proyección fuera muy complicada (las únicas proyecciones oficiales son las de usos del suelo futuro)

- Los modelos de reparto modal arrojaban resultados poco creíbles, como la preferencia modal de la población hacia el transporte público a igualdad de costes y tiempos con el vehículo privado.

En la figura siguiente se muestra la tasa de sondeo por zona de transporte (TAZ) para la zonificación utilizada ( 750 zonas), en la que se aprecia que hay pocas zonas con una muestra de más de 30 familias, y la existencia de grandes áreas con muestras muy bajas. 


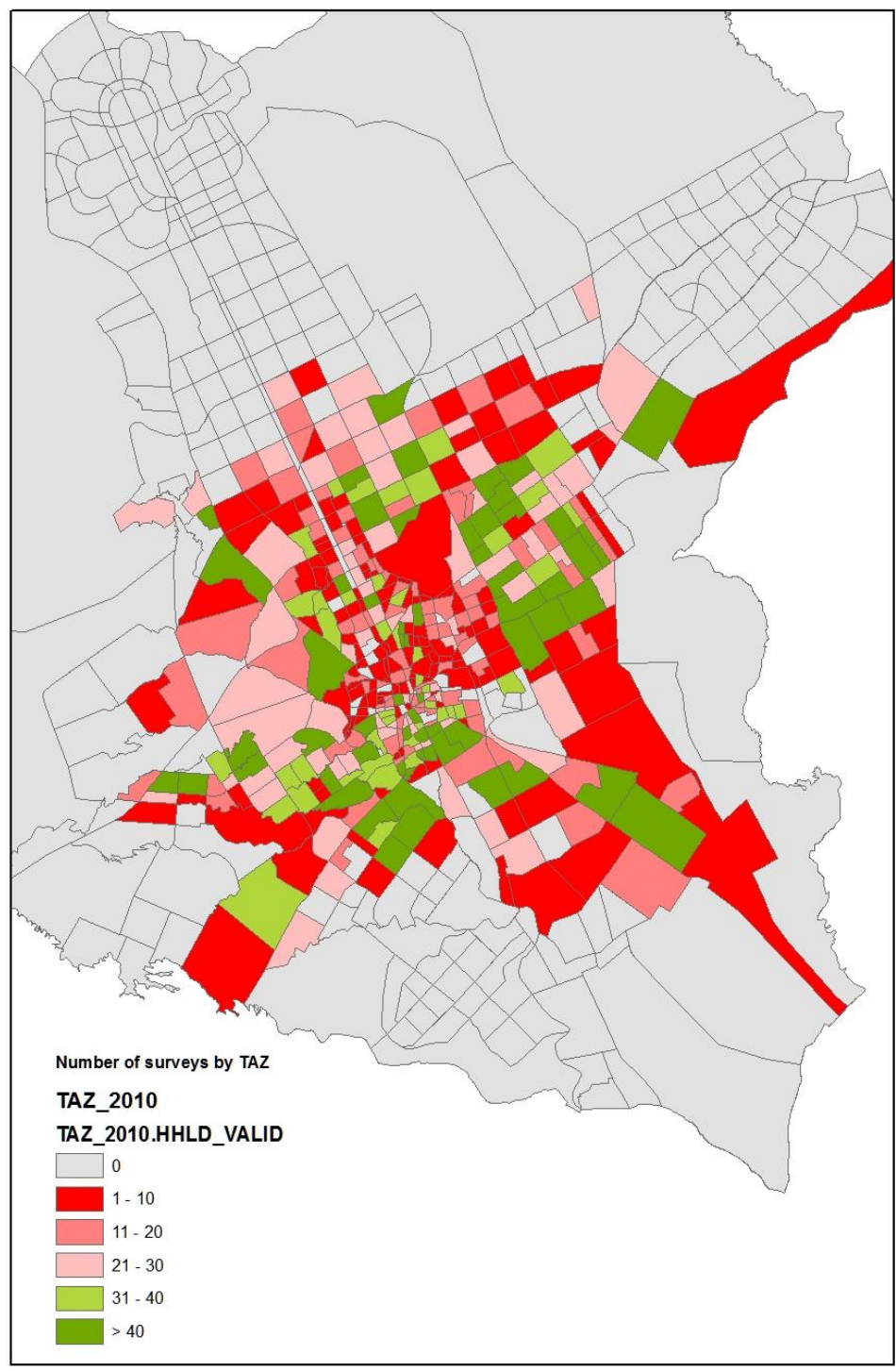

Figura 3 - Hogares encuestados por TAZ encuesta 2004

Los objetivos de la actualización de los modelos eran:

- Actualizar los modelos de producción/atracción y distribución espacial con una nueva EDM reducida; que explicara la movilidad actual de la ciudad con la mayor desagregación posible

- Construir un nuevo modelo de reparto modal, que permitiera obtener resultados razonables para la nueva oferta de transporte público, y que fuera sensible a las variables más relevantes

Las restricciones eran el presupuesto (que limitaba el número de encuestas), el plazo disponible y la información socioeconómica. No se disponía de un censo actualizado por TAZ (sólo datos agregados), y la única proyección oficial de la que se disponía eran los usos del suelo futuros. 


\section{PLANTEAMIENTO GENERAL DE LA ACTUALIZACIÓN DE LOS MODELOS DE DEMANDA}

Para actualizar los modelos de demanda se siguió el siguiente proceso:

- Analizar los resultados de la EDM 2004 para adoptar un criterio acerca de la información que sería útil recoger en la nueva EDM. En muchos casos, parte de la información anterior era irrelevante o no era significativa de cara a la modelización; y la simplificación de los cuestionarios permitía aumentar el número de encuestas. Esta simplificación se realizó manteniendo en lo posible la compatibilidad con los cuestionarios anteriores.

- Diseñar el proceso de toma de datos para la EDM: selección de las zonas a encuestar, número de encuestas por zona, proceso de encuesta, etc. Como criterio general, se seleccionaron las zonas infra- representadas en la EDM 2004 y las zonas de nuevo desarrollo.

- Diseñar una encuesta específica para los modelos de reparto modal, para lo que se realizaron entrevistas focales y encuestas de preferencias declaradas; y se ajustaron posteriormente modelos desagregados de elección de modo de transporte.

- Ajustar modelos de generación/atracción y distribución espacial a partir de los datos de la nueva EDM y otras fuentes disponibles.

- Incorporar los modelos en la plataforma EMME y crear los programas para evaluar diferentes configuraciones de la red y períodos temporales de análisis.

El trabajo de campo de las encuestas domiciliarias en Riad presenta algunas peculiaridades que se consideraron a la hora de diseñar el proceso de toma de datos. En general, no es posible acceder a los residentes en la vivienda si no está presente el cabeza de familia o un familiar responsable, lo que reduce el tiempo útil al día para realizar encuestas (sólo es posible por la tarde); y por algunas experiencias anteriores, se descartó la posibilidad de hacer encuestas telefónicas. Se puso particular cuidado en evitar los errores de las encuestas anteriores, por lo que se insistía en que los diarios de viajes debían ser rellenados por cada habitante del hogar, y no por el cabeza de familia. Además, muchos de los residentes no son árabes y su conocimiento del idioma es limitado, por lo que fue necesario emplear encuestadores bilingües que al menos dominaran una de lenguas de los grupos nacionales con más representación en Riad (paquistaníes e hindúes).

\subsection{Realización de la EDM 2014}

Una vez fijado el número de encuestas domiciliarias a realizar por TAZ, el proceso general que se siguió está esquematizado en la siguiente figura: 


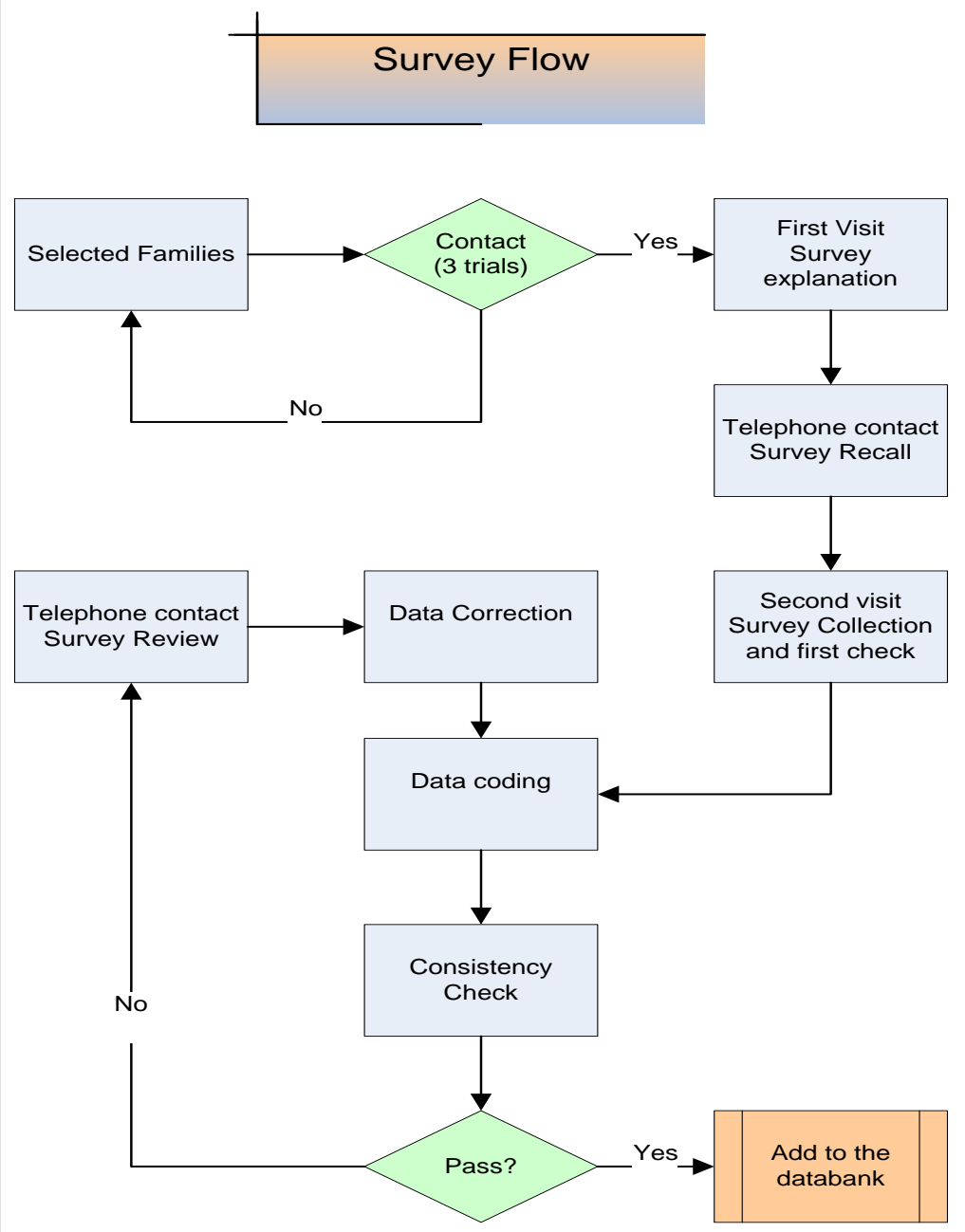

Figura 3 - Proceso de la EDM 2014

Por la falta de censos de población para este trabajo, la selección de las familias a encuestar se hizo por calles, intentando seleccionar en lo posible una muestra variada de tipos de familias, nacionalidades, etc.; para obtener una muestra lo menos sesgada posible. Se mantuvo un control estricto de los resultados que se iban obteniendo cada día, de manera que todos los días se analizaban algunos parámetros clave de las encuestas realizadas (como los viajes por persona y motivo, la consistencia de los datos, etc.) y en caso de que los valores fueran anómalos, los supervisores telefoneaban al domicilio de los encuestados para confirmar o corregir la información de los cuestionarios.

Simultáneamente se realizó una serie de encuestas de preferencias declaradas en lugares escogidos de la ciudad para obtener datos para los modelos de elección de modo. Previamente se habían realizado grupos focales con hombres y mujeres de diferentes nacionalidades y estratos sociales para obtener datos relevantes para el diseño de las encuestas de preferencias declaradas. 


\subsection{Resultados y ajuste de los modelos de demanda}

Finalmente se realizaron encuestas domiciliarias en 1534 hogares, con 7412 personas encuestadas (de las que sólo viajaron 5534, el 75\%) y se registraron 15673 viajes. El número de viajes por persona al día en la muestra se indica en la siguiente figura, en la que se puede observar que el bajo número de viajes impares al día es coherente con lo que se puede esperar a priori.

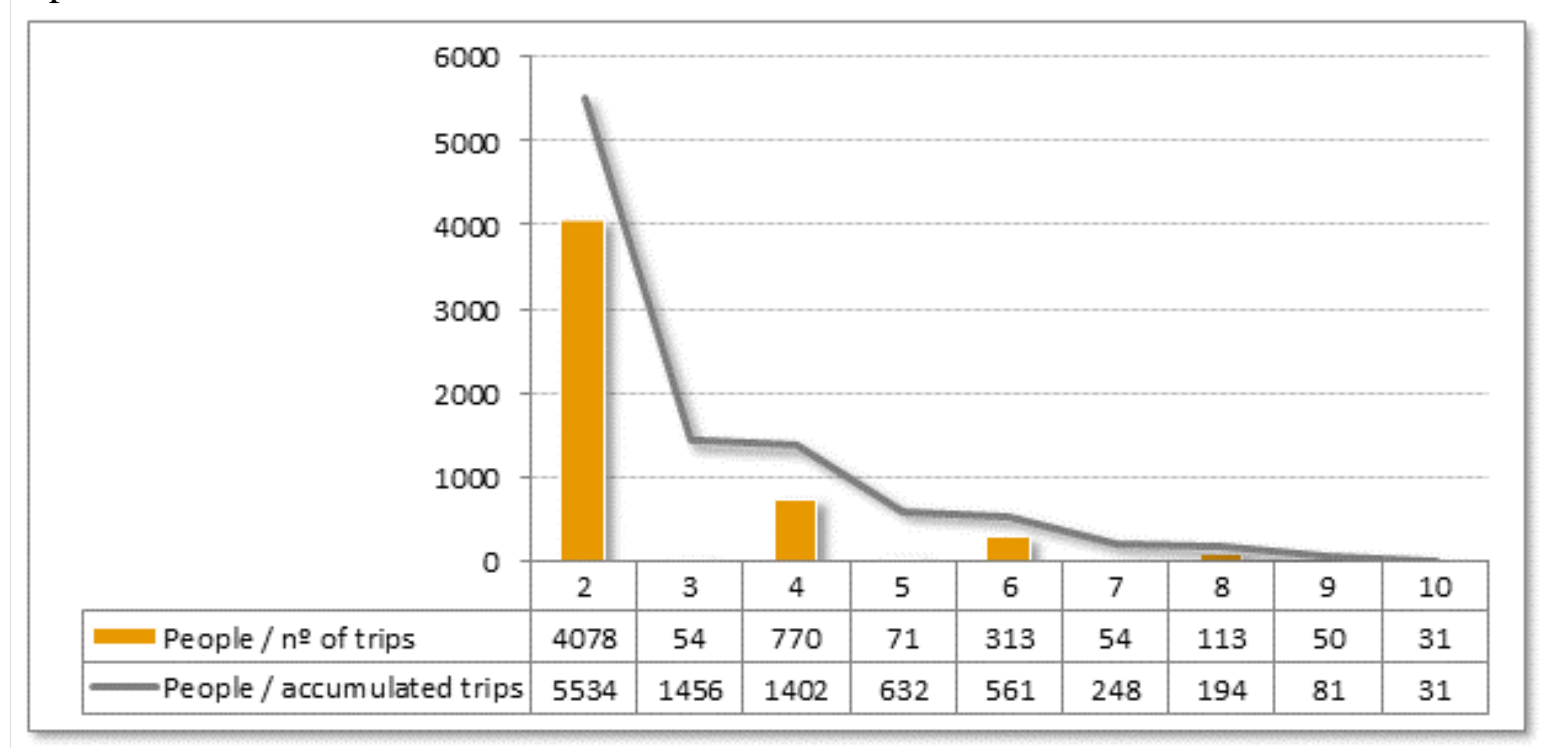

\section{Figura 3 - Número de viajes por persona EDM 2014}

En cuanto a los modos de transporte, el $85 \%$ de los viajes se realizan en vehículo privado, y sólo en el $1.5 \%$ de los viajes se utiliza el transporte público. Un porcentaje significativo (10.6\%) utiliza buses privados o de empresa o buses escolares para el transporte.

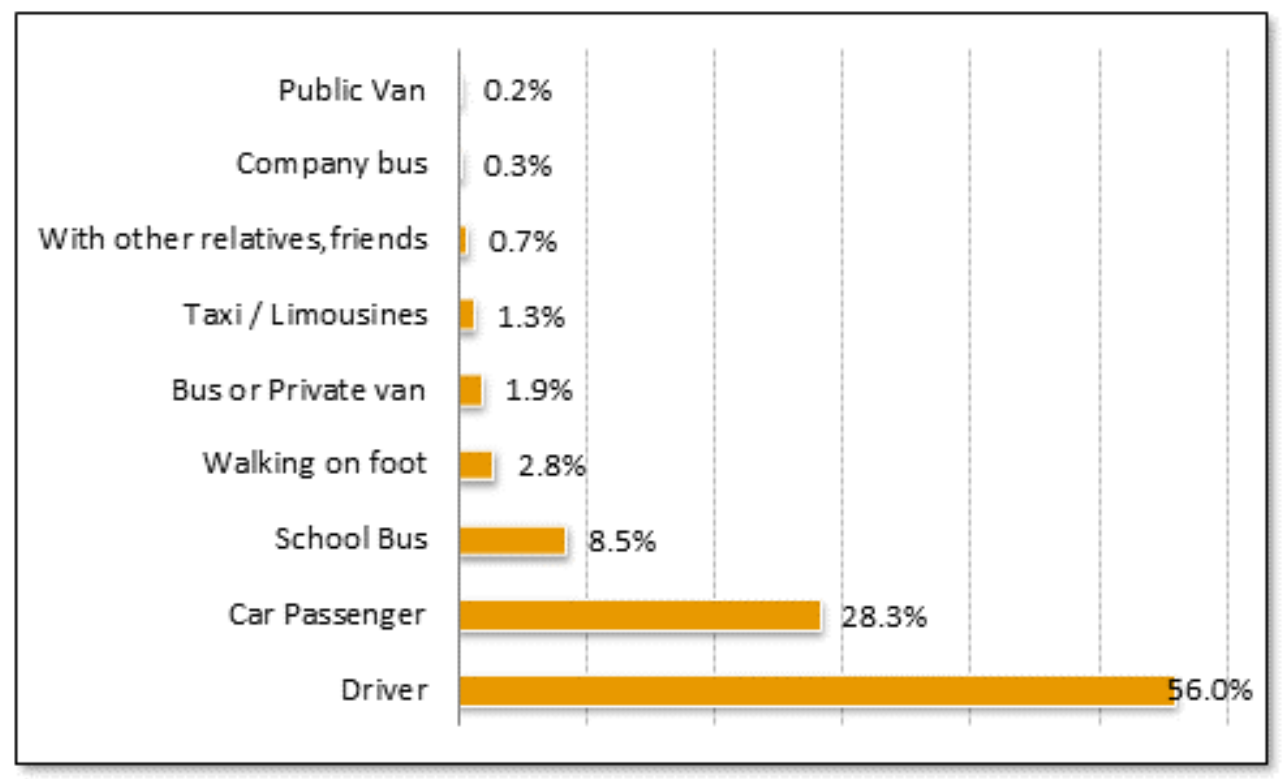

Figura 4 - Modos de transporte EDM 2014

Además de la información de viajes y socioeconómica recolectada en la EDM 2014, la 
explotación de los datos proporcionó una valiosa información para la selección inicial de las variables a incluir en los modelos de producción de viajes. Se realizó un análisis de varianza para dividir la muestra en grupos homogéneos de usuarios y tipos de viaje. En general se encontró que la nacionalidad era una variable relevante para la segmentación de la muestra; y en consecuencia se desarrollaron modelos diferentes para saudíes y no saudíes, porque presentaban comportamientos diferenciados; así como por motivos de viaje

\subsubsection{Modelos de producción de viajes}

Se han empleado regresiones lineales múltiples para el ajuste de los modelos de generación de viajes y se seleccionaron las variables con mayor correlación con el número de viajes observados. Se ajustaron modelos diferentes para:

- Población: Saudí y no saudí

- Motivo del viaje: trabajo (HBW), estudios (HBS), otros (HBO) y no basados en casa (NHB)

- Período del día: Hora punta de la mañana (AM) y todo el día.

\begin{tabular}{|c|l|l|c|r|}
\cline { 3 - 5 } \multicolumn{2}{c|}{} & \multicolumn{3}{c|}{ ALL DAY } \\
\hline Purpose & Variable & Nationality & Coefficient & \multicolumn{1}{c|}{$\mathrm{R}^{2}$} \\
\hline \multirow{2}{*}{ HBW } & \multirow{2}{*}{ Employed } & Saudi & 1.2544 & 0.78 \\
\cline { 3 - 5 } & & Non-Saudi & 2.0453 & 0.81 \\
\hline \multirow{2}{*}{ HBS } & \multirow{2}{*}{ Students } & Saudi & 1.8969 & 0.96 \\
\cline { 3 - 5 } & & Non-Saudi & 1.6394 & 0.86 \\
\hline \multirow{2}{*}{ HBO } & \multirow{2}{*}{ Adult+16 } & Saudi & 0.9011 & 0.62 \\
\cline { 3 - 5 } & & Non-Saudi & 0.8582 & 0.45 \\
\hline \multirow{2}{*}{ NHB } & \multirow{2}{*}{ Students } & Saudi & 0.3934 & 0.39 \\
\cline { 3 - 5 } & & Non-Saudi & 0.4235 & 0.23 \\
\hline
\end{tabular}

\begin{tabular}{|c|l|l|c|c|}
\cline { 3 - 5 } \multicolumn{2}{c|}{} & \multicolumn{3}{c|}{ MORNING PEAK (6-8) } \\
\hline Purpose & Variable & Nationality & Coefficient & $\mathrm{R}_{2}$ \\
\hline \multirow{2}{*}{ HBW } & \multirow{2}{*}{ Employed } & Saudi & 0.423 & 0.63 \\
\cline { 3 - 5 } & & Non-Saudi & 0.5116 & 0.57 \\
\hline \multirow{2}{*}{ HBS } & \multirow{2}{*}{ Students } & Saudi & 0.9046 & 0.94 \\
\cline { 3 - 5 } & & Non-Saudi & 0.8051 & 0.85 \\
\hline \multirow{2}{*}{ HBO } & \multirow{2}{*}{ Adult+16 } & Saudi & 0.2153 & 0.48 \\
\cline { 3 - 5 } & & Non-Saudi & 0.1791 & 0.29 \\
\hline \multirow{2}{*}{ NHB } & \multirow{2}{*}{ Students } & Saudi & 0.2079 & 0.39 \\
\cline { 3 - 5 } & & Non-Saudi & 0.1949 & 0.28 \\
\hline
\end{tabular}

Tabla 1 - Modelos de producción de viajes

\subsubsection{Modelos de atracción de viajes}

Los modelos de atracción de viajes dependen de la actividad en la zona de destino, es decir, de los viajes que atrae cada tipo de empleo, los comercios, los hospitales y centros médicos, las zonas de ocio, etc.

En Riad, como en muchos otros lugares, no hay información detallada sobre la localización de los puestos de trabajo, y la única información útil para el ajuste de los modelos de atracción son los usos del suelo, que se pueden dividir por TAZ para obtener la superficie dedicada a cada uso.

Sin embargo, la mayor dificultad para el ajuste de los modelos de atracción proviene de la muestra escogida: con una muestra pequeña, la representatividad de la información por zona de destino es muy baja; y por lo tanto no es conveniente ajustar modelos zonales a partir únicamente de estos datos.

Para solventar en parte este problema se procedió a:

- Añadir las encuestas de 2012 al banco de datos de 2014

- Identificar el número de personas por TAZ de acuerdo al sexo y la nacionalidad (saudí y no saudí) 
- Estimar un factor de expansión para cada categoría (TAZ_ sexo y nacionalidad)

- Esta expansión permite el cálculo de las variables explicativas de los modelos de generación para evitar el problema de los viajes no reportados en la EDM 2004.

- Aplicando los nuevos modelos de generación a las variables explicativas por TAZ se calcula el número de viajes producidos (AM y $\mathrm{PM}$ ); que resultaron considerablemente mayores que los reportados en 2004 por el crecimiento de población y por los viajes no incluidos en la EDM 2004.

- Finalmente, se obtienen los viajes atraídos por TAZ por
- Motivo del viaje
○ Población saudí y no saudí
○ Hombre y mujer

Con el cálculo de los viajes atraídos se ajustaron modelos de regresión para explicar la atracción de viajes, y se analizó la diferencia entre los viajes calculados por el modelo y los viajes obtenidos en la re-expansión de los datos como se ha indicado anteriormente, para aplicar factores correctores a algunas zonas. Los principales problemas observados se debían a zonas especiales en las que la densidad de empleos o puestos escolares era muy baja con respecto al resto de la ciudad, por lo que el número de viajes estimados por los modelos era mayor que el de la base de datos. Típicamente, estas zonas correspondían al aeropuerto, el distrito diplomático, algunas universidades, etc. Otras zonas especiales atraían más viajes que los estimados por el modelo, como algunos centros comerciales, el Ministerio de Trabajo (que atrae muchos viajes por gestiones), etc.

Se re-.estimaron los modelos excluyendo algunas zonas especiales para mejorar el ajuste, y el valor de los viajes atraídos por dichas zonas se sustituyó por el de la base de datos de las EDM. La estructura final de los modelos de producción atracción quedó como indica la siguiente figura (la estructura es idéntica para todo el día y para la hora punta de la mañana).Excepto en el caso de los viajes por motivo trabajo, para los que el modelo de atracción está segmentado por nacionalidades, se encontró que los modelos que producían un mejor ajuste eran los modelos en los que se consideraban iguales los viajes de saudíes y no saudíes. 


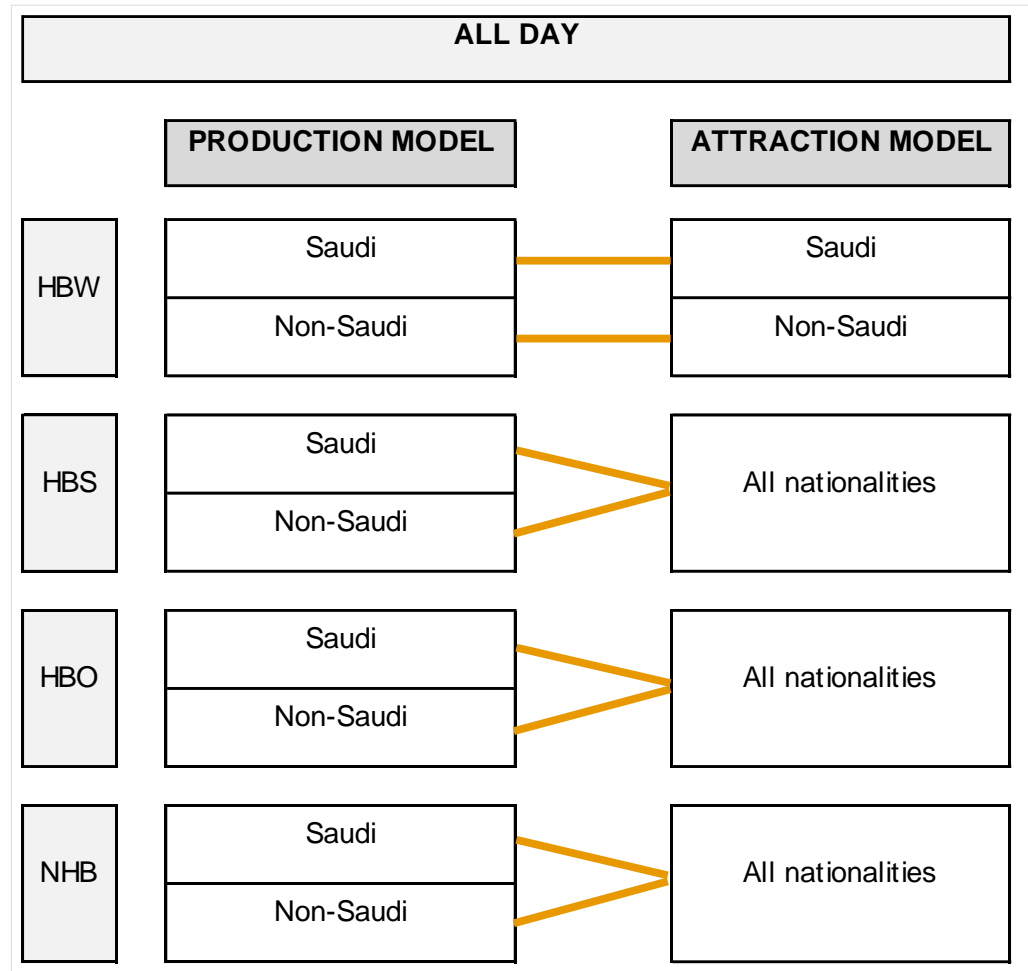

Figura 5 - Estructura de los modelos de generación / atracción

\subsubsection{Modelos de distribución espacial}

Para los modelos de distribución espacial se probaron distintas alternativas:

- Utilizar la misma formulación que el modelo de 2004

- Utilizar un modelo de gravedad ajustado con la nueva EDM

- Utilizar un modelo de balance tridimensional de matrices con el tiempo de viaje como variable de segmentación

- Utilizar un modelo de balance tridimensional de matrices con la distancia de viaje como variable de segmentación

Una vez desechadas las dos primeras alternativas por distintas razones, se analizó el impacto del incremento del número de viajes en los tiempos y las distancias de recorrido desde 2004 a 2013. En la siguiente tabla se muestran las diferencias para AM:

\begin{tabular}{|rrrrrrr|}
\hline Año & Autos AM & vh*km & vh*hora & $\begin{array}{c}\text { Tiempo } \\
\text { medio }(\mathrm{m})\end{array}$ & $\begin{array}{c}\text { Longitud } \\
\text { media }(\mathrm{km})\end{array}$ & $\begin{array}{r}\text { Velocidad } \\
\text { media } \\
(\mathrm{km} / \mathrm{h})\end{array}$ \\
\hline 2004 & 375118 & 4208271 & 123201 & 19.7 & 11.2 & 34.2 \\
2014 & 586240 & 7199578 & 300285 & 30.7 & 12.3 & 24 \\
\hline
\end{tabular}

Tabla 2 - Cambios en los tiempos y las longitudes de viaje, 2004-2014

Utilizar el tiempo de viaje como variable en el modelo de gravedad (la práctica usual) implicaría que el tiempo de viaje observado en 2014 debería ser aproximadamente el mismo que en 2004 (con un pequeño incremento). Si así fuera, y puesto que la velocidad media baja de 34 a $24 \mathrm{~km} / \mathrm{h}$; la longitud media de los viajes debería ser en 2014 de unos $7 \mathrm{~km}$ en lugar de los 12.3 observados, lo que no parece tener ninguna lógica. En ese caso, la reducción de 
viajes en AM sólo se podría explicar porque los usuarios encuentran trabajo junto a sus hogares o se cambian a lugares más próximos a su trabajo. Puesto que nada de eso parece haber sucedido, se escogió un modelo de balance tridimensional de matrices con la distancia de viaje como variable de segmentación; que se aplica de forma incremental (sólo para los nuevos viajes).

\subsubsection{Modelos de elección de modo}

Hacer un modelo de reparto modal para estimar la utilización de un nuevo modo de transporte púbico de calidad en Riad presenta dificultades considerables:

- El transporte público en Riad tiene una importancia marginal en el reparto total

- El transporte público actual presenta un estándar de calidad muy bajo, y es percibido por los usuarios como un modo de transporte para los usuarios de menor renta de la ciudad (en su mayoría trabajadores inmigrantes)

- Las mujeres no utilizan el transporte público, y hasta hoy, hay dudas sobre la autorización por parte de la Administración para que lo utilicen de forma generalizada.

- La inexistencia de impuestos que graven la posesión o el uso del automóvil, junto a los bajos precios del combustible hacen que sea difícil para los potenciales usuarios de transporte público plantearse siquiera la posibilidad de su uso.

Evidentemente, los datos actuales sirven de poco para el ajuste de un modelo de elección de modo. En los grupos focales, todos los participantes mostraban su rechazo a la utilización del transporte público actual como alternativa, incluso si ahorraban tiempo y dinero frente al viaje en coche. Por otro lado, con las encuestas de preferencias declaradas, en especial si se muestra de un modo irrealmente favorable el nuevo modo, se corre el riesgo contrario: los usuarios pueden mostrar una preferencia excesiva en las encuestas, pero seguirán sin cambiar su elección de modo cuando exista la nueva red de transporte público.

Obtener un balance realista no es sencillo; y además hay que tener en cuenta que no posible anticipar la calidad del servicio que finalmente ofrezca el nuevo modo; que será determínate en el caso de Riad para poder atraer demanda. Con la alternativa a seguir utilizando el vehículo privado con unos costes similares a los actuales; se corre el riego de que sólo sea atractiva para los usuarios actuales y para los trabajadores que ahora utilizan autobuses de empresa -porque las empresas ahorrarán costes y suprimirán los servicios si hay otra alternativa para que sus trabajadores lleguen al trabajo-.

Con este marco de referencia, se diseñó una encuesta de PD de elección binaria en la que se mostraba distintas alternativas de tiempos de viaje, costes, transbordos y tiempos a pie entre vehículo privado y transporte público; y entre distintos modos de transporte público; como se muestra en las siguientes figuras. 


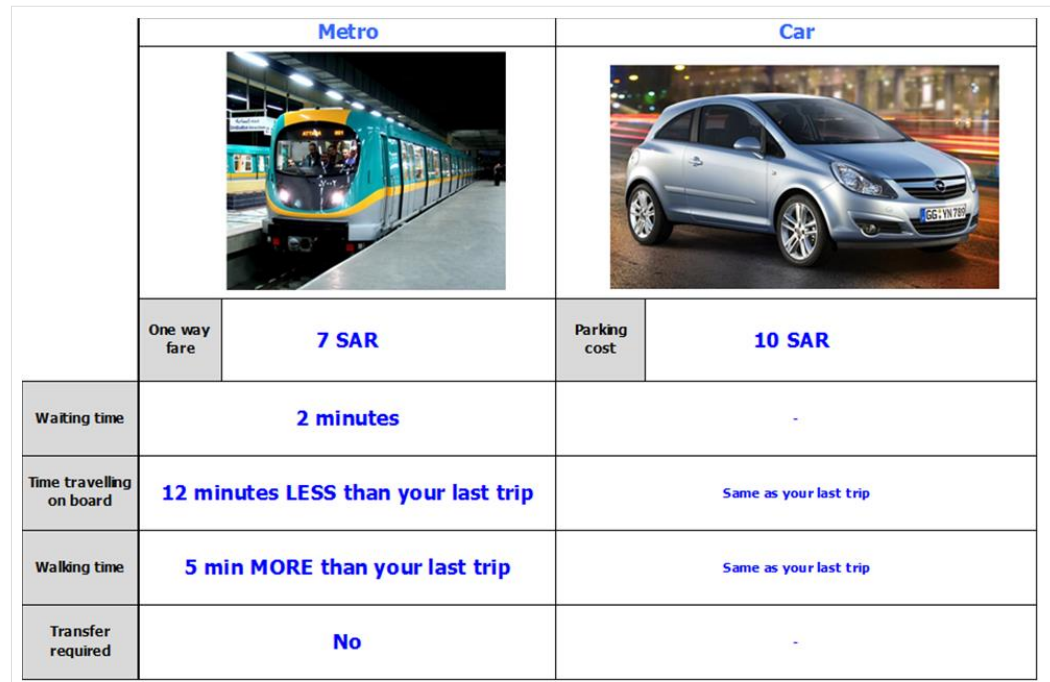

Figura 6- Ejemplo de elección metro- coche

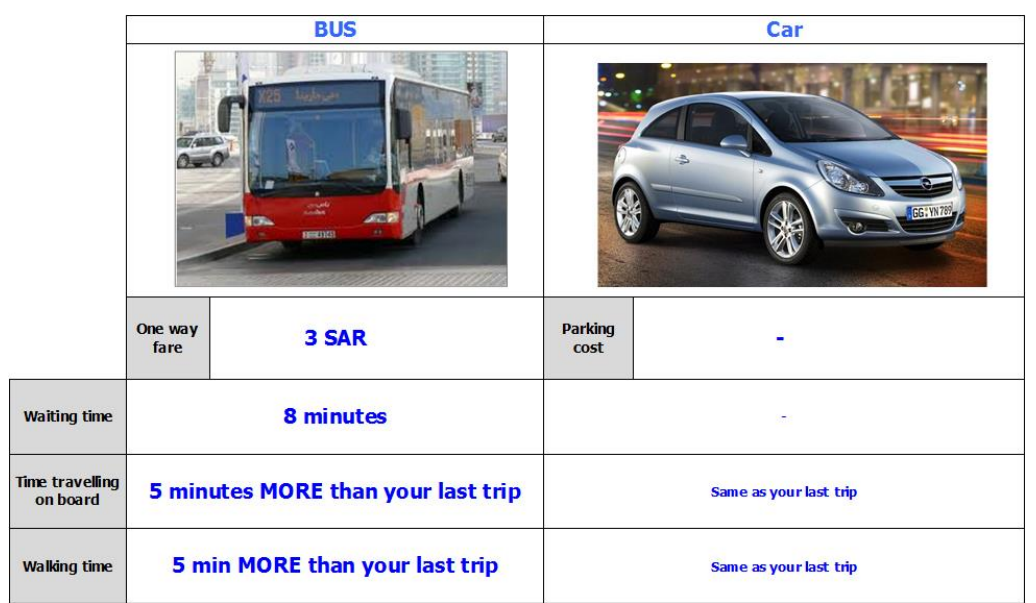

\section{Figura 7- Ejemplo de elección bus- coche}

\begin{tabular}{|c|c|c|c|c|}
\hline & \multicolumn{2}{|r|}{ Metro } & \multicolumn{2}{|r|}{ Taxi/ Limousine } \\
\hline & $\begin{array}{c}\text { One way } \\
\text { fare }\end{array}$ & 5 SAR & Taxi fare & Same as your last trip \\
\hline Warting time & & 2 minutes & & Same as your last trip \\
\hline $\begin{array}{l}\text { Time travelling } \\
\text { on board }\end{array}$ & $13 \mathrm{mi}$ & LESS than your last trip & & Same as your last trip \\
\hline Walling time & & 12 minutes & & Same as your last trip \\
\hline
\end{tabular}

Figura 8- Ejemplo de elección bus- coche 


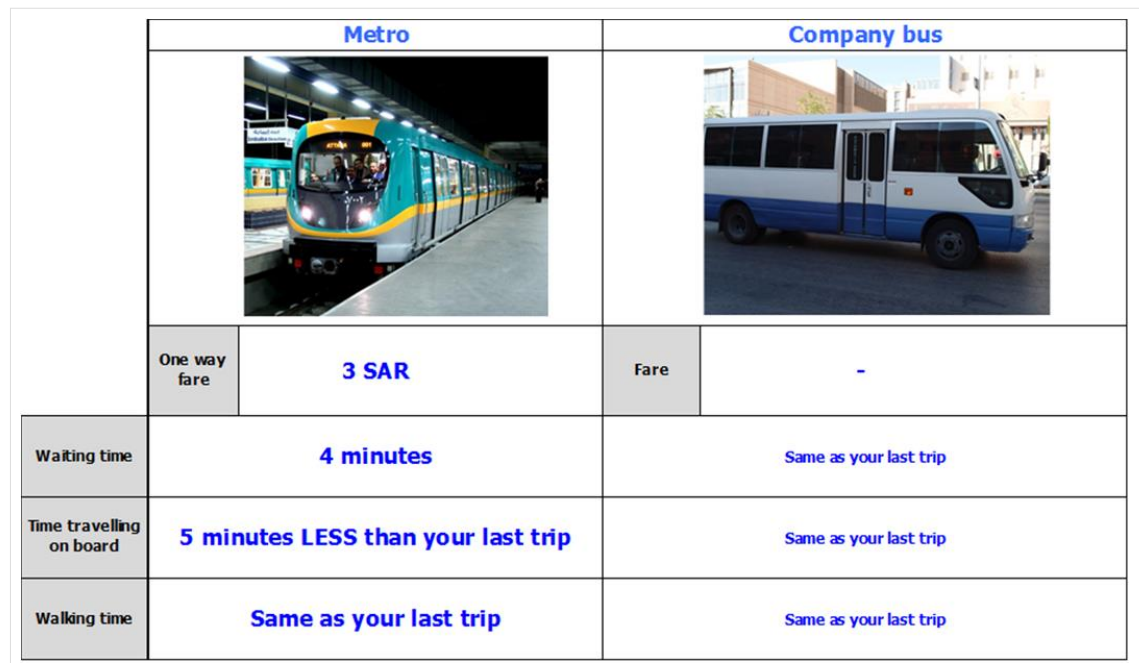

Figura 9- Ejemplo de elección bus- coche

Se ajustaron modelos diferentes para usuarios de coche saudíes y no saudíes, usuarios de taxi y usuarios de buses de empresa- Los resultados de los modelos de elección discreta ajustados (logit) son a priori consistentes y razonables, y están resumidos en las tablas siguientes.

\begin{tabular}{|l|rrrrr|}
\hline \multicolumn{6}{|c|}{ Usuarios de Coche } \\
\hline & $\begin{array}{c}\text { Valores económicos (SAR) } \\
\text { Segmento }\end{array}$ & $\begin{array}{c}\text { Transbordo } \\
\text { (SAR/m) }\end{array}$ & $\begin{array}{c}\text { Tiempo a } \\
\text { pie } \\
\text { (SAR) }\end{array}$ & $\begin{array}{c}\text { Tiempo de } \\
\text { espera } \\
(\mathrm{SAR} / \mathrm{m})\end{array}$ & $\begin{array}{c}\text { Constante } \\
\text { coche } \\
(\mathrm{SAR} / \mathrm{m})\end{array}$ \\
\hline Saudíes & 0.4 & 5.2 & 0.8 & 0.6 & 3.7 \\
No Saudíes & 0.9 & 13.6 & 1.8 & 1.5 & 5.1 \\
\hline
\end{tabular}

Tabla 3. Valores económicos usuarios de coche

\begin{tabular}{|l|rrrr|}
\hline \multicolumn{5}{|c|}{ Usuarios de taxi } \\
\cline { 2 - 5 } & $\begin{array}{c}\text { Viempo en } \\
\text { vehículo } \\
\text { Segmento }\end{array}$ & $\begin{array}{c}\text { Tiempo a } \\
\text { pie (SAR/m) }\end{array}$ & $\begin{array}{c}\text { Tiempo de } \\
\text { espera } \\
(\mathrm{SAR} / \mathrm{m})\end{array}$ & $\begin{array}{c}\text { Constante } \\
\text { taxi (SAR) }\end{array}$ \\
\hline Todos & 1.8 & 3.5 & 2.7 & 17.3 \\
\hline
\end{tabular}

Tabla 4. Valores económicos usuarios de taxi

\begin{tabular}{|l|ll|}
\hline \multicolumn{3}{|c|}{ Usuarios de bus de empresa } \\
\hline \multirow{4}{*}{} & Valores económicos (SAR) \\
\cline { 2 - 3 } & $\begin{array}{c}\text { Tiempo en } \\
\text { vehículo } \\
\text { (SAR/m) }\end{array}$ & $\begin{array}{c}\text { Cosntante } \\
\text { transporte } \\
\text { público } \\
\text { Segmento }\end{array}$ \\
\hline Todos & 0.2 & 1.8 \\
\hline
\end{tabular}

Tabla 5. Valores económicos usuarios bus empresa 


\section{CONCLUSIONES}

Como se muestra con el ejemplo de Riad, es posible actualizar modelos de demanda con datos reducidos, si las restricciones económicas o temporales no permiten hacer una encuesta domiciliaria completa. Las encuestas domiciliarias son caras y no siempre es posible hacerlas en las mejores condiciones, incluso aunque no existan restricciones presupuestarias.

Con datos reducidos, y si se va a utilizar un modelo clásico de cuatro etapas o alguna variante; los modelos de producción de viajes y de distribución espacial presentan pocos problemas para su ajuste. Con un conjunto de datos no muy grande se puede caracterizar la producción de viajes y las pautas de distribución de viajes en la ciudad, Sin embargo, el ajuste de un nuevo modelo de atracción puede ser mucho más complicado. El caso de Riad es un caso extremo, porque la EDM anterior tenía graves deficiencias, y ha obligado a utilizar un método un tanto complejo para poder obtener resultados. En un caso menos desfavorable, las complicaciones serán menores, pero en todo caso se debe analizar cuidadosamente las diferencias de información entre dos encuestas consecutivas.

En muchas ciudades, los modelos envejecen porque no se alimentan con nuevos datos que permitan incorporar los cambios en la estructura urbana o en las pautas de movilidad; y este envejecimiento es tanto mayor cuanto más pronunciados sean los cambios. Cambiar el paradigma de mantener los modelos estáticos durante 10 años hasta una nueva (y grande) EDM puede redundar en mejores modelos, un entendimiento más cabal de las pautas de los cambios en los patrones de viajes y mejores resultados en la prognosis y en la evaluación de proyectos.

Las EDM son por su naturaleza complejas y plantean problemas que no siempre se pueden corregir en el momento de su realización: problemas con algunos cuestionarios, subrepresentación de determinados tipos de viaje, problemas relacionados con el propio método de encuesta (por ejemplo, las encuestas telefónicas), etc. Un proceso continuo de actualización mediante un número reducido de nuevas encuestas permite solventar esos problemas en ejercicios consecutivos, en lugar de esperar 10 o más años hasta otro nuevo ejercicio (que seguramente presentará errores nuevos).

Por último, señalar que las encuestas domiciliarias no son la única fuente disponible para la actualización de modelos de demanda. Datos de los operadores de transporte público, aforos, datos de tráfico telefónico, etc.; se pueden combinar con los datos de las EDM’s para mejorar las estimaciones; aunque no siempre es una tarea sencilla incorporar datos de fuentes diferentes. 


\section{AGRADECIMIENTOS}

Este trabajo ha sido el fruto de la participación de numerosas personas, tanto de la Administración de Riad (ADA) como de las empresas que han participado en su desarrollo. Quiero agradecer especialmente el apoyo del Dr. Jalal Nafakh y de Turki Suleiman de ADA, del Dr. Gunnar Landfester, del Dr. Ton Voge y de Mohammed Mujeebullah Khan, de Dornier; y por supuesto del resto del equipo de Diadro: Rocío Morales, Pilar Escáriz y Susana Santamaría. 